\title{
Production de rayons $X$ par interaction laser femtoseconde - agrégats de gaz rare
}

\author{
E. Lamour, D. Vernhet, J.-P. Rozet, S. Dobosz ${ }^{1}$, M. Lezius ${ }^{1}$, M. Schmidt ${ }^{1}$, \\ D. Normand ${ }^{1}$, J.-C. Gauthier ${ }^{2}$, S. Jacquemot ${ }^{3}$, M. Cornille ${ }^{4}$ et J. Dubau ${ }^{4}$
}

\author{
GPS, UMR 75-88 du CNRS, Universités Paris 7 et Paris 6, 75251 Paris cedex 05, France \\ ${ }^{1}$ CEA Saclay, DSM/DRECAM/SPAM, bâtiment 522, 91191 Gif-sur-Yvette cedex, France \\ 2 LULI, UMR 76-05 du CNRS, École Polytechnique, 91128 Palaiseau cedex, France \\ ${ }^{3}$ CEA-DAM/lle de France, BP. 12, 91680 Bruyères-le-Châtel, France \\ ${ }^{4}$ DARC, Observatoire de Paris, place Jules Janssen, 92195 Meudon cedex, France
}

\begin{abstract}
Résumé: Nous avons réalisé toute une série de mesures sur la production de rayons X émis par des agrégats (Ar) $(\mathrm{Kr})_{\mathrm{n}}$ et $(\mathrm{Xe})_{\mathrm{n}}$ (avec $\mathrm{n}$ compris entre $10^{4}$ et $10^{6}$ atomes/agrégats) soumis à des impulsions laser intense $\left(\leq 10^{18}\right.$ $\mathrm{W} / \mathrm{cm}^{2}$ ) ultracourte (entre 80 et $800 \mathrm{fs}$ ). Nous avons déterminé l'énergie des photons émis ainsi que le taux d'émission absolu en fonction de certains paramètres physiques régissant l'interaction : taille et numéro atomique des agrégats, intensité au pic du laser. Compte tenu du fort taux absolu d'X d'émis, nous avons également effectué des mesures par spectroscopie $\mathrm{X}$ de haute résolution dans le cas des agrégats d'Ar. Nous avons ainsi mis en évidence ta production d'ions très chargés (de $11+$ à $16+$ ) ayant une lacune en couche $\mathrm{K}$. De plus, les résultats indiquent que le rayonnement $\mathrm{X}$ est émis avant l'explosion des agrégats sur une échelle de temps de quelques $100 \mathrm{fs}$.
\end{abstract}

\section{INTRODUCTION}

Des études expérimentales ont montré que l'interaction laser intense-agrégats de gaz rares générait une excitation électronique très intense de la matière. En particulier, elles ont mis en évidence la génération d'ions multichargés rapides d'énergies atteignant le MeV [1-4], ainsi que des électrons "chauds" avec des

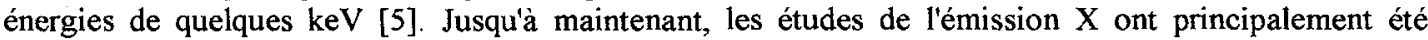
limitées à des observations qualitatives et à l'élaboration de modèles controversés à l'heure actuelle [6-8]. C'est dans ce cadre que nous avons effectué des mesures sur la production de rayons $X$ émis par des agrégats $d^{\prime}(\mathrm{Ar})_{n}$, de $(\mathrm{Kr})_{\mathrm{n}}$ et de $(\mathrm{Xe})_{\mathrm{n}}$ (avec $\mathrm{n}$ compris entre $10^{4}$ et $10^{6}$ atomes/agrégats) « soumis » à des impulsions laser dans le domaine infra rouge $(790 \mathrm{~nm})$, d'une durée comprise entre 80 et 800 fs et d'une énergie pouvant atteindre $90 \mathrm{~mJ}$ (correspondant à des éclairements $\leq 10^{18} \mathrm{~W} / \mathrm{cm}^{2}$ ). Ces expériences ont été réalisées auprès du laser LUCA du SPAM/DRECAM à Saclay. En dehors des applications potentielles (i.e. sources de rayons $\mathrm{X}$ ), les motivations premières de ces études sont de caractériser les mécanismes impliqués dans la création des nanoplasmas chauds induits lors de l'interaction et tout particulièrement d'étudier les processus de chauffage des électrons de la matière irradiée. L'analyse des spectres $\mathrm{X}$, à basse et haute résolution, permet de déterminer l'évolution de population des états excités des ions multichargés créés en fonction de certains paramètres physiques régissant l'interaction : taille moyenne, densité et numéro atomique des agrégats; intensité au pic en ce qui concerne le laser. Ici, nous présenterons très brièvement les résultats les plus significatifs.

\section{RESULTATS}

Dans un premier temps, par la détermination de l'énergie des photons émis, nous avons caractérisé le type de lacunes créées pour chaque type d'agrégats ainsi que l'état de charge moyen des ions émetteurs : production de lacune $\mathrm{K}$ pour l'Ar, lacunes L pour le $\mathrm{Kr}$ [9] et le Xe. Pour chaque type d'agrégats, le tableau 1 donne les résultats obtenus pour une pression amont du jet d'agrégats de 18 bars ( $\equiv$ densité atomique de $910^{14} \mathrm{~cm}^{-3}$ dans la zone d'interaction) et une intensité du pic laser de $510^{17} \mathrm{Wcm}^{-2}$. Dans ces conditions, le taux d'émission $\mathrm{X}$ absolu a été trouvé maximum pour les agrégats de $\mathrm{Kr}$, soit de l'ordre de $10^{6}$ photons/pulse. On peut donc s'attendre à un taux d'émission $\mathrm{d}^{\prime} \mathrm{X}$ de $2 \mathrm{keV}$ de $10^{11}$ photons/pulse (!) en travaillant avec des densités atomiques $10^{5}$ fois plus grandes. Il est intéressant de noter aussi que l'état de charge moyen observé est plus grand que ceux déduits des mesures de temps de vol des ions après 
explosion des agrégats (par exemple, des ions $\mathrm{Ar}^{10+}$ au maximum ont été détectés par cette méthode dans des conditions similaires).

Tableau 1 : Energies des photons, taux absolus d'émission $\mathrm{X}$ et état de charge moyen des ions émetteurs.

\begin{tabular}{lccc}
\hline & argon & krypton & xénon \\
\hline $\mathrm{E}_{\text {photon }}(\mathrm{eV})$ & 3086 & $1741 / 2248$ & 4109 \\
$\mathrm{~N}_{\text {photons/pulse }}$ & $710^{4}$ & $410^{6}$ & $1,510^{5}$ \\
$\langle\mathrm{Q}\rangle$ & 14 & $24-27$ & $31-35$ \\
\hline
\end{tabular}

Compte tenu du taux d'X émis, nous avons effectué des mesures par spectroscopie $\mathrm{X}$ de haute résolution et de grande transmission dans le cas des agrégats d'Ar. Dans ce cas "simple", pour lequel les configurations électroniques sont bien connues, les spectres enregistrés peuvent être interprétés, sans ambiguïté, par la production d'états excités $1 s 2 p 2 \ell^{\mathrm{n}}$ d'ions fortement multichargés (11+ jusqu'à 16+), ayant une lacune en couche $K$. Une analyse plus précise indique, au moins pour les ions d'états de charge les plus élevés $(15+$ et $16+)$, que le rayonnement $\mathrm{X}$ est émis avant l'explosion des agrégats sur une échelle de temps $\leq 100$ fs. Cette émission donne donc des informations directes sur la dynamique du nanoplasma chaud créé lors de l'interaction, la génération d'ions multichargés énergétiques étant quant à elle reliée aussi à la dynamique de l'explosion des agrégats. Plusieurs de ces spectres ont été obtenus dans des conditions différentes afin de réaliser deux types d'études complètement : une en fonction de la pression amont $\left(\mathrm{P}_{0}\right)$ c'est-à-dire de la taille des agrégats (taille $\left.\propto \mathrm{P}_{0}^{2}\right)$ et l'autre en fonction de l'intensité $\left(\mathrm{I}_{\mathrm{pic}}\right)$ du laser. Ces études ont permis d'établir les lois d'échelle suivantes pour le taux absolu d'émission $\mathrm{X}$ :

$$
\mathrm{N}_{\text {photons } / \text { pulse }} \propto \mathrm{I}_{\text {pic }}^{3 / 2} \text {.et } \mathrm{N}_{\text {photons/pulse }} \propto \mathrm{P}_{0}^{5 / 3}
$$

\section{DISCUSSION ET PERSPECTIVE}

L'ensemble des résultats obtenus met en évidence l'importance des mécanismes de collisions (électronsions) à l'intérieur d'un agrégat unique dans la création de lacune en couche interne et permet d'établir les premières bases d'une modélisation à même d'expliquer l'interaction. Une première comparaison avec un modèle simple basé sur ces mécanismes d'ionisation des atomes de l'agrégat par des électrons [10] permet d'interpréter quantitativement les taux absolus de photons mesurés. La confrontation avec des calculs plus complexes, utilisant des fonctions de distributions électroniques de type thermique et monocinétique, nous permet d'ores et déjà, par ailleurs, d'avoir une estimation de la densité ionique des nanoplasmas créés, ainsi que de la température et de la densité électronique au moment de l'émission $X$.

Les résultats montrent que le rayon moyen de l'agrégat au moment de l'émission est 6 fois plus grand que son rayon initial. Des études complémentaires sont planifiées, et les résultats seront discutés en détail dans un article à venir.

\section{Références}

1. Purnell et al., Chem. Phys. Lett. 229 (1994)

2. Snyder et al., Phys. Rev. Lett. 77 (1996)

3. Lezius et al., J. Phys. B : At. Mol. Phys. 30 (1997)

4. Ditmire et al., Nature 386 (1997)

5. Shao et al., Phys. Rev. Lett. 77 (1996)

6. McPherson et al., Nature 370 (1994)

7. Rose-Petruck et al., Phys. Rev. A 55 (1997)

8. Ditmire et al., Phys. Rev. A 53, (1996); Ditmire et al., J. Phys. B : At. Mol. Phys. 31 (1998)

9. Dobosz et al., Phys. Rev. A 56 (1997)

10. Rozet et al. Physica Scripta à paraître (2000) 